\title{
Study of the cyclone separator for waste plastics by Taguchi method
}

\author{
Xianhai Yang ${ }^{1, a^{*}}$, Xiang Cheng ${ }^{1, b}$ Xia Liu $^{1, c}$ and Lupeng Song ${ }^{1, d}$
}

${ }^{1}$ School of Mechanical Engineering, Shandong University of Technology, Zibo, 255049, China

ayangxhsdut@163.com, bhappychengx@163.com, cx-mf@tom.com, ${ }^{\mathrm{d}}$ wyhightech@163.com

Keywords: Cyclone separator, Waste plastic sorting, Taguchi method, Orthogonal experiment

\begin{abstract}
The separating principle of the cyclone separator has been analyzed by analyzing the tangential speed, the axial speed, and the pressure distribution of the flow field inside the cyclone separator. The dimensional parameters of the separator with larger effects on the separation efficiency of plastics have been identified such as the length of the overflow pipe, the length of the separator, and the diameter of the discharge opening. Based on Taguchi method, orthogonal experiments have been designed for separating process parameters according to the identified dimensional parameters. Furthermore, the separating process has been simulated for different dimensional parameters and different operating conditions. The separating efficiency of waste plastics has been obtained. According the analyses, the optimization of the separator has been conducted. The optimal dimensional parameters and operating parameters of the cyclone separator have been identified for high efficient separation of waste plastics.
\end{abstract}

\section{Introduction}

Pollutions introduced by widely used plastic products become more and more serious ${ }^{[1,2]}$. Conventionally, waste plastics are buried or burned ${ }^{[3]}$. These methods have progressed a lot, but still there are secondary pollutions to generate harmful gas ${ }^{[4]}$. Therefore, the best way to handle waste plastics is to recycle them. But in the recycling process, it becomes more and more important to sort these waste plastics with various densities ${ }^{[5]}$. Drum screens have been used to sort municipal wastes ${ }^{[6]}$. But drum screens are not suitable for the sorting of waste plastics with various densities. In previous pneumatic sorting experiments, the wind directions are always fixed resulting in the limited contacting time between the plastic particles and the blowing air. Consequently, the sorting efficiency is very low. The cyclone separating method has been applied and introduced in this paper. The dimensions of the sorting equipment have been reduced largely. The contacting time between the plastic particles and the blowing air and the sorting efficiency have been increased.

\section{Cyclone sorting principle}

There are balanced guide model and residence time model for cyclone sorting. Previous experiments show that the Barth model of the balanced guide models is relatively better than other models. As shown in Fig. 1, the Barth model forms a hypothesis plane CS inside the separator by extending the overflow pipe to the discharge opening of the separator.

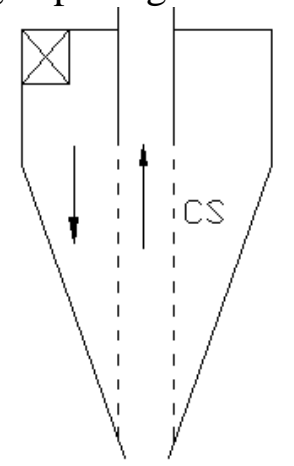

Fig. 1 the hypothesis plane CS 
In the plane CS, the centrifugal force bearing by the plastic particles and the air-flow resistance come to a balance.

$$
\rho_{t} \frac{\pi d_{t}^{3}}{6} \frac{v_{\theta C S}^{2}}{0.5 D}=3 \pi \mu d_{t} v_{r C S}
$$

Where, $\rho_{t}$ is the density of plastic particles, $d_{t}$ is the diameter of plastic particles, $v_{\theta C S}$ is the tangential speed in the plane CS of plastic particles, $v_{r C S}$ is the axial speed in the plane CS of plastic particles, $\mathrm{D}$ is the diameter of the cyclone separator.

The centrifugal force bearing by the plastic particles is in direct proportion to the mass of the plastic particles. Assume diameters of plastic particles are the same, the centrifugal force bearing by the plastic particles is in direct proportion to the density of the plastic particles. The mixed waste plastic particles enter the entrance of the separator under the air forces. With the constraints of the side wall, motions of waste plastic particles change from linear to revolving by the centrifugal actions. Most of the airflows move in a spiral way and enter into the cone portion of the separator. The formed vortex is known as the outside vortex. Meantime, mixed waste plastics in the airflow with larger densities will contact and collide with the side wall of the separator by centrifugal forces. Then, these plastic particles will fall into the discharge opening following the outside vortex and leave the separator. Under the motions of the outside vortex, high pressure area and low pressure area will be formed near the outside wall and in the central area of the separator, respectively. Therefore, air flows up at the center of the separator and plastic particles with lower densities will fly up and escape from the overflow pipe. Based on this principle as shown in Fig. 2, waste plastic particle with various densities are sorted.

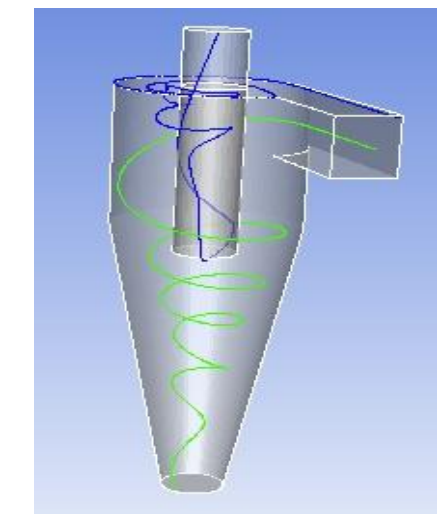

Fig. 2 The schematic view of the cyclone separator

\section{The inside flow field analysis}

The key parameters affecting the sorting efficiency can be identified by analyzing the air field inside the separator.

Speed analysis of the flow field. The speed vectors are shown in Fig. 3, where the transverse and longitudinal cross sections are shown. It shows that the air forms the downward vortex firstly and then forms the upward motions at the bottom inside the cyclone separator. The air core cylinder has been formed at the center of the separator. The air flow is relatively symmetrical. The symmetry at the upper area is worse than that at the lower area of the separator. It can be explained that the asymmetry is mainly caused by the single entrance design of the separator.

The flow field has the feature of double-layer flow as the downward flow at the outside area and the upward flow at the inside area. Furthermore, there exist many secondary vortexes.

The formulae of the outside vortex and the inside vortex are as Eq. 1 and Eq. 2, respectively.

$$
d_{50}=\sqrt{\frac{14.33 a b \mu}{\pi \rho H v}}
$$




$$
\operatorname{Re}=\frac{d v \rho}{\mu}
$$

Where, $m$ and $n$ are vortex indexes and their values are from 0.5 to 0.7 decided by the size of the separator, $c_{1}$ and $c_{2}$ are constant, $\mathrm{r}$ is the axial distance.

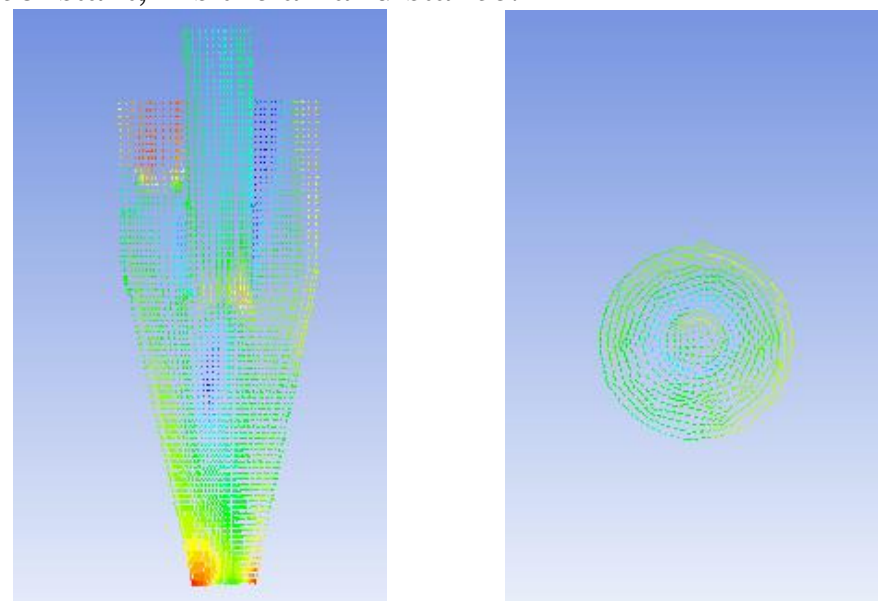

Fig. 3 Speed vectors inside the separator

The analyses show that the length of the overflow pipe has more important effects on the sorting performances.

Fig. 4 shows the cloud distributions of the transverse and longitudinal cross sections of the axial speed. It can be seen that plane CS is the separating plane of plastic particles with different densities. The diameter of the discharge opening has larger effects on the separating efficiency.
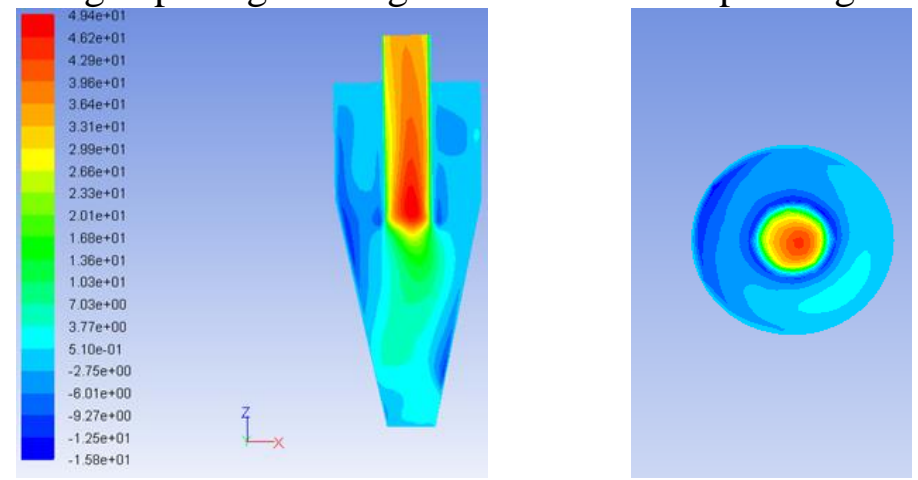

Fig. 4 Axial speed distributions

Pressure decrease analyses. There are two pressure decrease areas inside the separator. One is from the entrance to the interface between the outside vortex and the inside vortex. Another is from the interface to the overflow opening. The distributions of the general pressure and the static pressure are shown in Fig. 5.
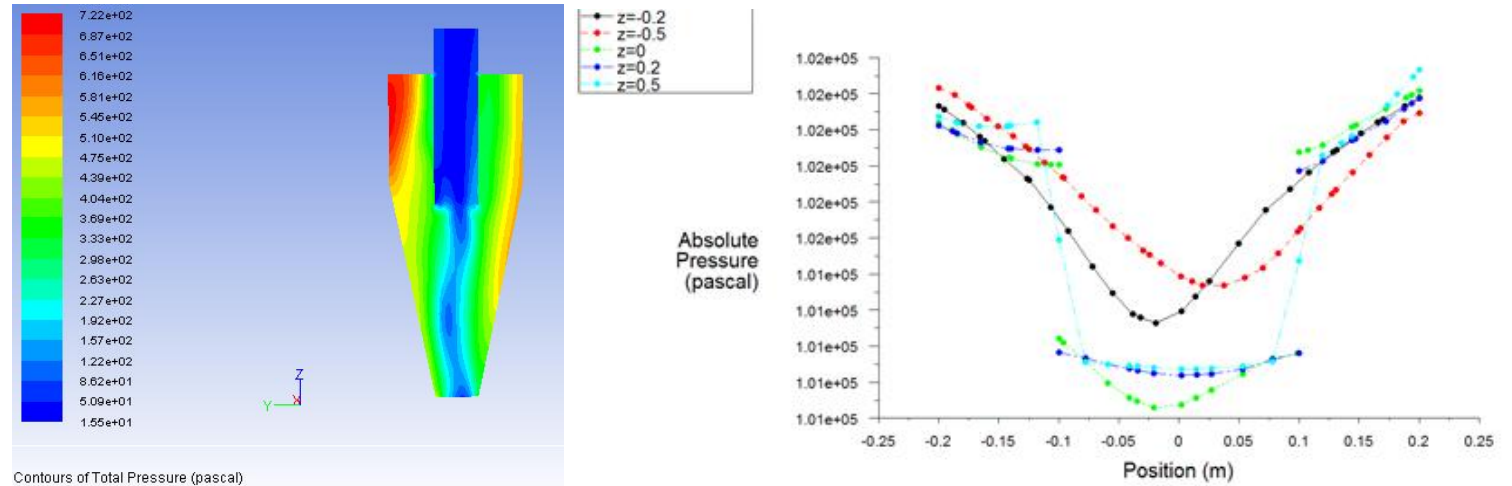

(a) The general pressure distribution 

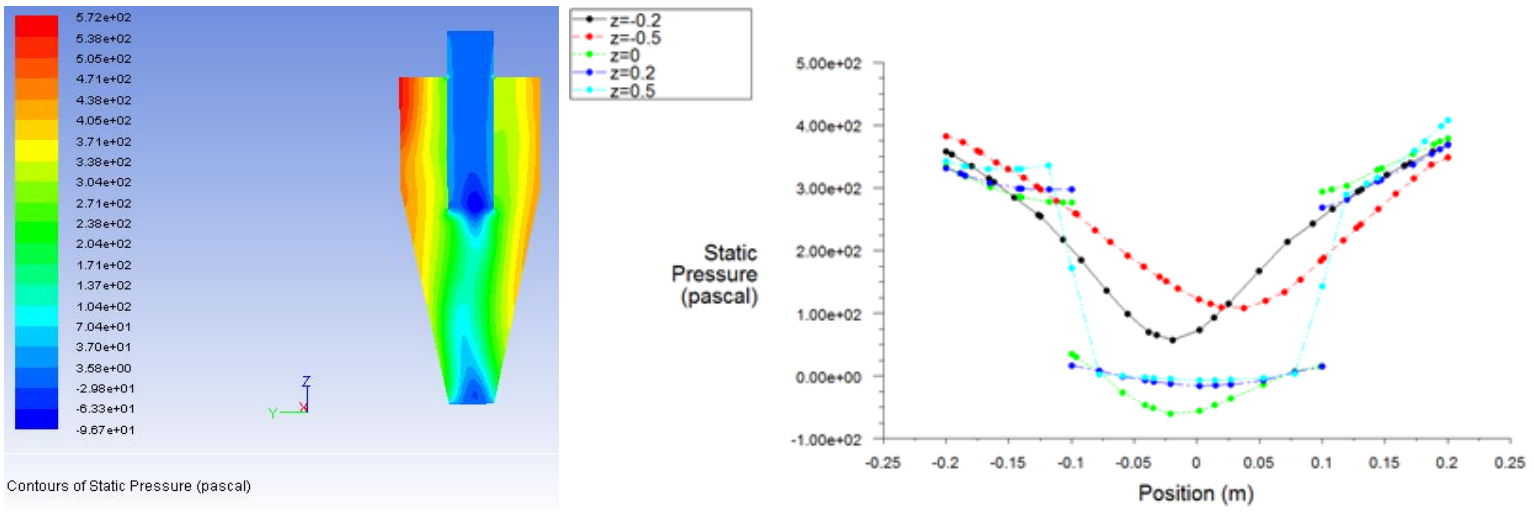

(b) The static pressure distribution

Fig. 5 Pressure distributions

From Fig. 5, the general pressure and the static pressure decrease very quickly with the reduction of the diameter. The static pressure of both the entrance and the overflow pipe are higher than that of the central area.

According to the above analyses, key parameters affecting the separating effects are the length of the overflow pipe, the length of the separator, and the diameter of the discharge opening.

\section{Experimental simulations}

Power spinning turbulent flow exists inside the separator. Therefore, the anisotropic RSM turbulent flow mode is adopted. The differential model adopts the QUICK model and the pressure compensation model adopts the PRESTO model. The Euler dual-fluids model is adopted. Air media works as the basic phase. Two different kinds of waste plastic particles work as the secondary and third phases, respectively. The collisions among plastic particles are neglected. Based on the above selections and the non-interlaced mesh SIMPLEC, the simulation model has been created. Fig. 6 shows the simulation results.

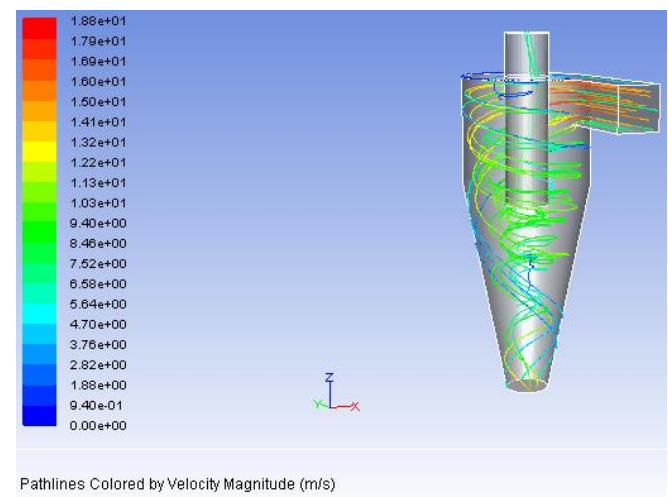

(a) Trajectories of the PET particle

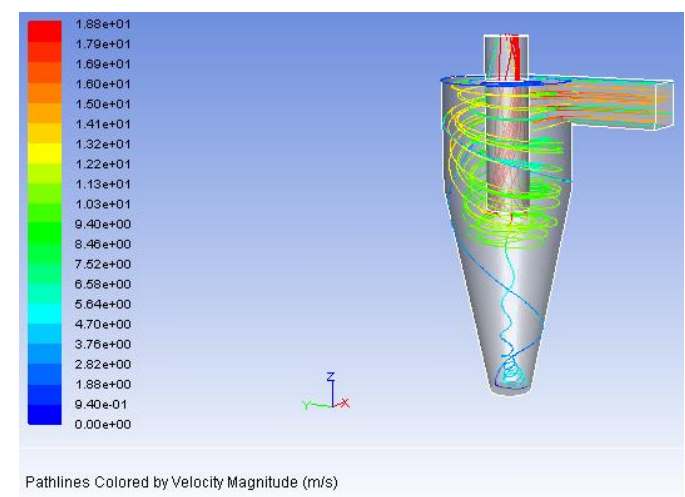

(b) Trajectories of the PE particle

Fig. 6 Simulation results

From Fig. 6, most of the PET plastic particles with larger density escape from the discharge opening. But there also some particles moves outside from the overflow opening. Because PE particles have a smaller density, the following performance of the air flow is better and the PE particles move outside from the overflow opening. That means the sorting of various waste plastic particles has been successfully achieved.

Experimental simulation results. Based on the above simulation model and the Taguchi method, the orthogonal experiments $\mathrm{L}_{25}\left(5^{5}\right)$ have been designed and conducted. Table 1 shows the experimental simulation results, where $Y_{1}$ and $Y_{2}$ are the separating efficiencies of the waste plastics PET and PS, respectively. 
Table 1 Experimental simulation results

\begin{tabular}{|c|c|c|c|c|c|c|c|}
\hline No. & $\begin{array}{c}\text { Length of } \\
\text { the } \\
\text { overflow } \\
\text { pipe (mm) }\end{array}$ & $\begin{array}{c}\text { Length of } \\
\text { the } \\
\text { separator } \\
(\mathrm{mm})\end{array}$ & $\begin{array}{c}\text { Diameter of } \\
\text { the discharge } \\
\text { openning } \\
(\mathrm{mm})\end{array}$ & $\begin{array}{c}\text { Speed at } \\
\text { the } \\
\text { entrance } \\
(\mathrm{m} / \mathrm{s})\end{array}$ & $\begin{array}{c}\text { Plastic } \\
\text { size } \\
(\mathrm{mm})\end{array}$ & $\mathrm{Y}_{1}$ & $\mathrm{Y}_{2}$ \\
\hline 1 & 100 & 1200 & 60 & 17 & 1.0 & 0.25 & 0.19 \\
\hline 2 & 100 & 1300 & 80 & 18 & 1.5 & 0.35 & 0.31 \\
\hline 3 & 100 & 1400 & 100 & 19 & 2.0 & 0.30 & 0.21 \\
\hline 4 & 100 & 1500 & 120 & 20 & 2.5 & 0.21 & 0.17 \\
\hline 5 & 100 & 1600 & 140 & 21 & 3.0 & 0.22 & 0.14 \\
\hline 6 & 200 & 1200 & 80 & 19 & 2.5 & 0.36 & 0.32 \\
\hline 7 & 200 & 1300 & 100 & 20 & 3.0 & 0.33 & 0.31 \\
\hline 8 & 200 & 1400 & 120 & 21 & 1.0 & 0.30 & 0.29 \\
\hline 9 & 200 & 1500 & 140 & 17 & 1.5 & 0.24 & 0.25 \\
\hline 10 & 200 & 1600 & 60 & 18 & 2.0 & 0.33 & 0.24 \\
\hline 11 & 300 & 1200 & 100 & 21 & 1.5 & 0.39 & 0.35 \\
\hline 12 & 300 & 1300 & 120 & 17 & 2.0 & 0.35 & 0.29 \\
\hline 13 & 300 & 1400 & 140 & 18 & 2.5 & 0.31 & 0.37 \\
\hline 14 & 300 & 1500 & 60 & 19 & 3.0 & 0.52 & 0.49 \\
\hline 15 & 300 & 1600 & 80 & 20 & 3.0 & 0.54 & 0.48 \\
\hline 16 & 400 & 1200 & 120 & 18 & 3.0 & 0.56 & 0.55 \\
\hline 17 & 400 & 1300 & 140 & 19 & 1.0 & 0.48 & 0.49 \\
\hline 18 & 400 & 1400 & 60 & 20 & 1.5 & 0.88 & 0.84 \\
\hline 19 & 400 & 1500 & 80 & 21 & 2.0 & 0.91 & 0.87 \\
\hline 20 & 400 & 1600 & 100 & 17 & 2.5 & 0.87 & 0.87 \\
\hline 21 & 500 & 1200 & 140 & 20 & 2.0 & 0.63 & 0.69 \\
\hline 22 & 500 & 1300 & 60 & 21 & 2.5 & 0.74 & 0.91 \\
\hline 23 & 500 & 1400 & 80 & 17 & 3.0 & 0.93 & 0.88 \\
\hline 24 & 500 & 1500 & 100 & 18 & 1.0 & 0.92 & 0.82 \\
\hline 25 & 500 & 1600 & 120 & 19 & 1.5 & 0.83 & 0.83 \\
\hline
\end{tabular}

Experimental analyses. From Table 1, the length of the overflow pipe has the greatest effects on the separating efficiency, which has the direct proportion to the length of the overflow pipe. The reason is that the short circuit flow at the bottom of the overflow opening reduces with the increase of the length of the overflow pipe. This kinds of effects become intensively when the density of the waste plastics becomes smaller and smaller. But it is not the smaller the best since chatter will happen when the separator runs at a higher speed because the air flow along the overflow pipe will have a shock effect on the pipe. The frequent chatter will cause the pipe break resulting in the air leakage. Moreover, it will result in the crest broken. Therefore, the length of the overflow pipe should be selected considering various actual conditions.

The secondary more important factor is the diameter of the discharge opening. It can be seen from Table 1 that the separating efficiency becomes lower when the diameter becomes smaller. The reason is that the item to be separated is waste plastics. The concentration of the waste plastic particles at the discharge opening becomes too large to cause larger frictions and possible ionizations. But when the diameter becomes too large, the separating efficiency also becomes smaller. The reason is that the interface between the outside and inside vortexes will be affected. The CS plane is too close to the inside wall to cause excessive frictions. 
With the increase of the length of the separator, the sorting efficiency increases at the beginning. But when the length of the separator reaches $1600 \mathrm{~mm}$, the sorting efficiency decreases. The reason is that the airflow changes to the overflow pipe before it reaches the discharge opening.

Based on the above analyses, the optimum parameters are $A_{5} B_{4} C_{2} D_{3} E_{2}$, namely, the length of the separator is $1500 \mathrm{~mm}$, the length of the overflow pipe is $500 \mathrm{~mm}$, the diameter of the discharge opening is $80 \mathrm{~mm}$, the air speed at the entrance is $17 \mathrm{~m} / \mathrm{s}$, and the size of the waste plastic particle is $2 \mathrm{~mm}$.

\section{Summary}

The separating model of waste plastics inside the cyclone separator has been introduced according to the separating principle analyses. Aiming at a higher sorting efficiency, simulation models have been created and key parameters affecting the efficiency have been identified as the length of the separator, the length of the overflow pipe, the diameter of the discharge opening. Based on Taguchi method, further experimental simulations have been conducted and optimum parameters have been achieved to form a solid basis for future design and development of high efficient waste plastic sorting equipments.

\section{Acknowledgement}

The authors wish to express their appreciation for the financial support by the Natural Science Foundation of Shandong Province with the grant numbers of ZR2013EEM011 and ZR2014EEM046.

\section{References}

[1] F. Long, B. Song, Q.H. Wang, X.F. Xia, L.L. Xue, Scenarios simulation on municipal plastic waste generation of different functional areas of Beijing, J. Mater. Cycles Waste Manag., 14 (2012) 250-258.

[2] S.H. Peng, L.Q. Chen, L.B. Li, M.Q. Xie, H.J. Huang, X.D. Liu, Debromination of flame-retarded TV housing plastic waste, J. Mater. Cycles Waste Manag., 12 (2010) 103-107.

[3] J.F. He, Y.M. Zhao, Z.F. Luo, C.L. Duan, L.H. Ge, Distribution characteristic of bed density in air dense medium fluidized bed based on the Euler-Euler model, J China Coal Society, 38 (2013) 1277-1282.

[4] L.Y. Wu, J. Li, F. Zhu, Study on the cyclone separating techniques of municipal rubbish, POPs forum 2012, Beijing, 278-280.

[5 ] X.H. Yang, C.Y. Lu, Kinematic properties and test of plastics' optimal separation equipment. Chinese Journal of Mechanical Engineering, 43(2007) 132-135.

[6] B. Li, Z.Y. Dong, Y.C. Zhao, Q.H. Zheng, J.L. Zhu, Study on the mechanical separation characteristics for municipal solid waste using trommel screener, Acta Sci. Circumstantiae, 31 (2011) 2268-2274. 